\title{
Protein comparison at the domain architecture level
} Byungwook Lee ${ }^{* 1}$ and Doheon Lee*2

\author{
Addresses: ${ }^{1}$ Korean BioInformation Center, KRIBB, Daejeon 305-806, Korea and ${ }^{2}$ Department of Bio and Brain Engineering, KAIST, \\ Daejeon 305-701, Korea \\ E-mail: Byungwook Lee* - bulee@kribb.re.kr; Doheon Lee* - dhlee@biosoft.kaist.ac.kr \\ ${ }^{*}$ Corresponding author
}

from Asia Pacific Bioinformatics Network (APBioNet) Eighth International Conference on Bioinformatics (InCoB2009)

Singapore 7-II September 2009

Published: 3 December 2009

BMC Bioinformatics 2009, 10(Suppl I5):S5 doi: 10.1186/147I-2105-10-SI5-S5

This article is available from: http://www.biomedcentral.com/I47I-2/05/I0/SI5/S5

(C) 2009 Lee and Lee; licensee BioMed Central Ltd.

This is an open access article distributed under the terms of the Creative Commons Attribution License (http://creativecommons.org/licenses/by/2.0), which permits unrestricted use, distribution, and reproduction in any medium, provided the original work is properly cited.

\section{Background}

Homology identification is part of a broad spectrum of genomic analyses, including the annotation of new whole genome sequences, the construction of comparative maps, the analysis of whole genome duplications and comparative approaches to identifying regulatory motifs [1]. The general method used to determine the function of newly discovered proteins is 
to transfer annotation from well-characterized homologous proteins sharing a common ancestry [2]. Current methods for the identification of homologous proteins can be largely classified into sequence-based and domain-based approaches [3]. Sequence comparison methods, such as BLAST and FASTA, are the commonly-used traditional approaches to identify homologous genes $[4,5]$. These methods assume that sequences with significant similarity share common ancestry, i.e. are homologs. However, the existence of multi-domain proteins and complex evolutionary mechanisms poses difficulties for sequence-based methods [6].

Domain-based methods use information of the domains contained in proteins [7]. Domains are the building blocks of all proteins, and present one of the most useful levels at which protein function can be understood [8]. Although the concept of a 'domain' now permeates biological descriptions, there are several definitions directed at different levels of the protein [9]. In structural biology, a domain is defined as a spatially distinct, compact and stable protein structural unit that could conceivably fold and function in isolation. Domains are also defined as distinct regions of protein sequence that are highly conserved throughout evolution. These are described as sequence homologs and are often present in different molecular contexts. Sequence-based domain definitions represent one of the most convenient and practically important levels at which the evolution and function of both proteins and domains can be understood.

Domain-based approaches identify homologous proteins generally by comparing protein domain architecture, which is the linear order of the individual domains in multidomain protein. About two thirds of proteins in prokaryotes and $80 \%$ of proteins in eukaryotes are multidomain proteins [10]. Studies of domain-based methods indicate that comparing domain architecture is a useful method for classifying evolutionarily related proteins and detecting evolutionarily distant homologs [11]. Several studies have proposed tools for domain architecture comparison, such as CDART [12] and PDART [9]. However, these methods are challenged by large families defined by 'promiscuous' (or 'mobile') domains, which combine in many ways with other domains to form different proteins [13]. Promiscuous domains have typically auxiliary functions to the primary role of protein, acting as signal transducers, or adapters $[14,15]$. They also play a major role in creating diversity of protein domain architecture in the proteome [16]. Because they are not directly related by homology, they should be given less importance in homology identification than key domains. Another problem inherent to these domain-based measures is that they treat all proteins in a domain architecture equally. They cannot discriminate among proteins belonging to the same domain architecture. Since most domain architectures consist of many proteins, identification methods are needed to determine which protein is the most homologous to the query protein within a set of proteins belonging to the same domain architecture.

Here we present a measure, called Weighed Domain Architecture Comparison (WDAC), of domain architecture similarity, which can be used to identify homologs of multidomain proteins. The key ideas are the use of weight scores for domain promiscuity and combining domain architecture comparison with sequence similarity method. The weight scores are calculated based on a domain's frequency and versatility in RefSeq [17] proteins. The effectiveness of our method is evaluated using human and nematode proteomes. We developed a web-based server to allow users to compare their proteins with protein domain architectures. The server is available at http://wdac.kr/.

\section{Methods}

\section{Domain assignment}

In this study we used the Pfam [18] database to analyze the domain organization of proteins. Pfam is a large and widely used database of protein domains and families. Pfam contains curated multiple sequence alignments for each family, as well as profile hidden Markov models for finding these domains in new sequences. Pfam also provides better genomic coverage than structure-based domain assignments, such as CATH [19] and SCOP [20], particularly for membrane proteins.

\section{Measuring the strength of domain promiscuity}

To measure the strength of domain promiscuity, we considered two features of protein domains, the first of which is domain abundance. Compared to non-promiscuous domains, promiscuous domains appear in many proteins because they are needed to perform auxiliary functions. Vogel et al. [21] have shown that the combination tendencies of domains can be explained by a random evolutionary process model, in which a highly abundant domain tends to form more combinations. To measure the abundance of a domain, we defined the Inverse Abundance Frequency (IAF). The basic concept of IAF is derived from the Inverse Document Frequency (IDF), a statistic commonly used in information retrieval. IDF is a measure based on the observation that a word that occurs in very few documents is more likely to differentiate between subjects than a word that occurs frequently [22]. Namely, IDF is a measure of the general importance of 
a term. The IDF score is obtained by dividing the number of all documents by the number of documents containing the term, and then taking the logarithm of that quotient. For example, if 'cow' appears in 100 documents out of a total of 10,000 and 'bovine' in 10 documents, the IDF scores of 'cow' and 'bovine' are 0.2 and 0.1 , respectively. Thus, the word ' $\mathrm{cow}^{\prime}$ conveys less information about the subject of the document than the word 'bovine'. The number of documents containing a term and the number of documents in the corpus are analogous to the proteins containing a domain and the total number of proteins under study in the IAF statistic, respectively. The definition of IAF for a domain, $d$, is

$$
\operatorname{IAF}(d)=\log _{2} \frac{p_{t}}{p_{d}}
$$

where $p_{t}$ is the number of total proteins and $p_{d}$ is the number of proteins containing domain $d$.

The second feature of protein domains that we consider is domain versatility. Promiscuous domains occurring in many protein clusters have many partner domain families while highly conserved domains appear in a small number of protein clusters and their neighbor domains are also conserved during evolution [16]. Thus, domains with the same abundance could have a different number of distinct partner domain families. To measure the versatility of a domain, we defined the Inverse Versatility (IV) obtained from the inverse of the number of distinct partner domain families at the $\mathrm{N}$ - and $\mathrm{C}$-sides adjacent to a domain. The definition of the IV of a domain, $d$, is

$$
I V(d)=\frac{1}{f_{d}}
$$

where $f_{d}$ is the number of distinct domain families adjacent to domain $d$. The weight score of a domain is simply calculated by the product of the IDF and the IV of a domain. Let us consider the theoretical example (Figure 1), where both domain A and domain B occur three times. Domain A has four distinct neighbors and domain $\mathrm{B}$ has only one distinct neighbor. Since the weight score of domain $\mathrm{A}$ is lower than that of domain $\mathrm{B}$, domain $\mathrm{A}$ is more promiscuous than domain $\mathrm{B}$.

\section{Comparison of domain architectures using weight scores} Using the domain weight scores, we compared domain architectures. First, the shared distinct domain families are compared. We represented the two sets of domains derived from two architectures as the indices, which are built using the vector-space model (VSM) [23]. Domain architectures were converted into a vector in which each component corresponds to the weight score of a domain. The similarity of the two vectors is measured by

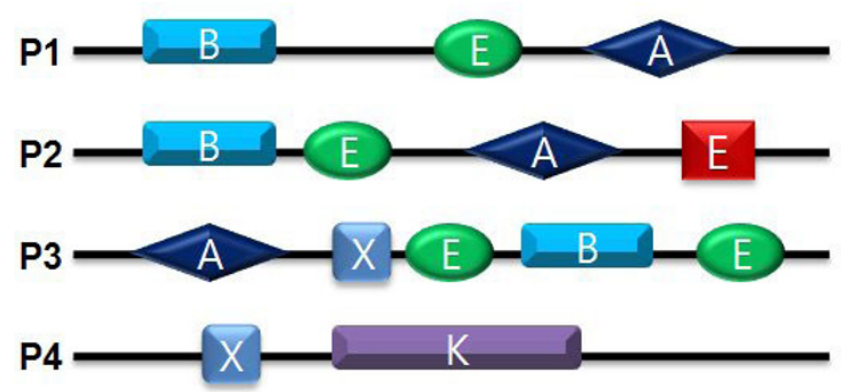

\section{Figure I}

Example calculation of the weight scores of domains. Black lines represent protein sequences and the colored boxes, circles, and diamonds represent different domains. The number of occurrences of two exemplary domains, A and $B$, is three (PI, P2, and $P 3)$, and the number of distinct partner domains of $A$ and $B$ is three $(E, D$, and $A)$ and one (E), respectively. The IAF scores of $A$ and $B$ are 0.125 , and the IV scores of $A$ and $B$ are 0.3 and I.0, respectively. We can obtain the final weight score of domain $A(0.038)$ and domain B (0.125) from the IDF and IV scores. From the weight scores of the two domains, we can determine that domain $\mathrm{A}$ is a more promiscuous domain than domain $\mathrm{B}$.

determining their cosine similarity, a measure based on the angle between two vectors (commonly used in text mining algorithms). If $x$ and $y$ are vectors of two domain architectures $X$ and $Y$, the cosine similarity is defined

$$
\operatorname{sim}(X, Y)=\frac{\sum_{k=1}^{n} x_{k} y_{k}}{\sqrt{\sum_{k=1}^{n} x_{k}^{2}} \times \sqrt{\sum_{k=1}^{n} y_{k}^{2}}} .
$$

The range of the cosine similarity is $[0,1]$, where 1 indicates that $x$ and $y$ have the same domains and 0 indicates that they share no domains.

Second, domain orders were considered. To measure the order similarity, we compared shared domain pairs between two domain architectures. In domain evolution, two- or three-domain combinations, called supradomain, are re-used in different protein context, and domain pairs in protein domain architecture occur in only one order, with only about $2 \%$ of such pairs occurring on both possible orders. The order similarity is measured by dividing the shared domain pairs $(Q s)$ by the total domain pairs $(Q t)$. The function is defined by

$$
\operatorname{order}(X, Y)=\frac{Q_{s}}{Q_{t}}
$$

The final similarity score between two domain architectures, $X$ and $Y$, is obtained by combining the indices from equations 3 and 4 (each normalized to $[0,1]$ ) using a simple linear function. 


\section{Pipeline for domain architecture comparison}

We constructed an automatic pipeline for identifying homologs of proteins (Figure 2). The pipeline programs were written in Perl and consist of four main steps. First, the pipeline assigns Pfam domains to a query protein and extracts a domain architecture from the Pfam annotation. Second, the query domain architectures are compared against the domain architecture database. Third, the query proteins are compared with RefSeq proteins using BLASTP [24]. Lastly, matched domain architectures and BLAST results are combined and sorted according to their similarity scores.

\section{Web-based server}

We developed a web-based server to provide a back-end pipeline for protein homology and to allow users to compare their protein sequences with a domain architecture database. The web interface is implemented with static HTML and CGI scripts, and MySQL DBMS is used to store the database.

\section{Results and discussion}

\section{Obtaining weight scores of protein domains}

We downloaded 6,042,750 protein sequences from the RefSeq database (Release 32). The domain content of the sequences was analyzed with Pfam 23.0 containing

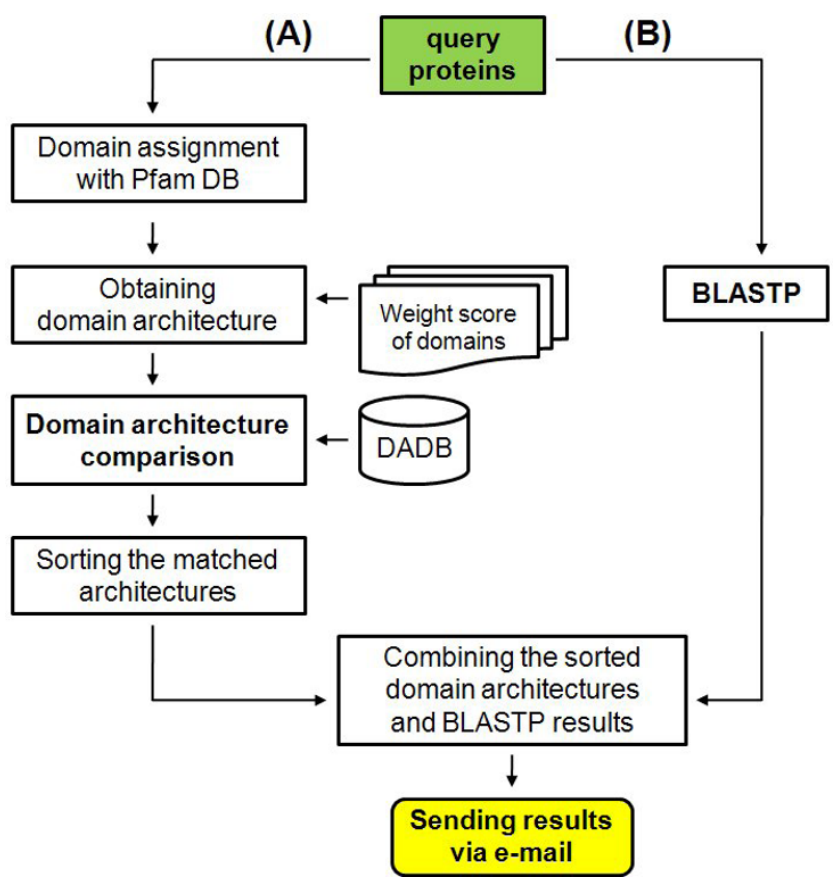

Figure 2

Workflow for the identification of protein homology. The pipeline combines sequence similarity information and domain architecture comparison methods.
10,340 families. The Pfam domain annotations of all RefSeq proteins were obtained from the Similarity Matrix of Proteins (SIMAP) [25] database. We filtered domain hits in proteins with a cutoff E-value of 0.01 and excluded proteins without Pfam signatures. We extracted all the Pfam domains from the Pfam-annotated proteins

Of the 6,042,750 RefSeq proteins, 3,942,678 (65\%) contain more than one Pfam domain. These Pfamannotated proteins were converted into domain architectures, in which we obtained 55,841 distinct domain architectures. The domain architecture data show that $90 \%$ of the domain architectures are kingdom-specific. Thus, we classified the 55,841 domain architectures into three kingdoms: Eukaryote, Bacteria, and Archaea (Figure 3). From these domain architectures, we extracted 8,775 domains and then divided them into the three kingdoms, where $17 \%$ of all the domains are common to all three kingdoms of life whereas $54 \%$ appear only in one kingdom.

Because domains are differently distributed in the three kingdoms and some domains are absent or present in one or two kingdoms, we assigned three kingdomspecific weight scores to each domain based on its abundance and versatility in the three kingdoms. To measure domain abundance, we obtained the kingdomspecific protein frequency for each domain. Most domains occur in a hundred or fewer proteins, but a

\section{Eukaryote Bacteria}

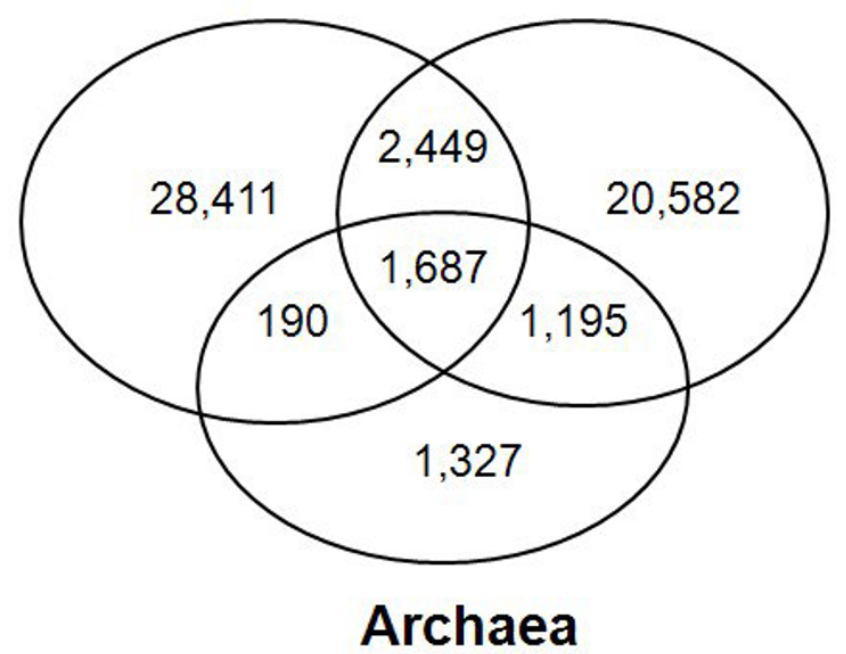

Figure 3

The distribution of domain architectures across the three kingdoms of life. Ninety percent of the domain architectures are kingdom-specific. 
few domains are highly duplicated and occur in over 10,000 sequences. The most abundant domain in the three kingdoms is ABC_tran (PF00005), appearing in 54,980 bacterial proteins. To measure domain versatility, we obtained kingdom-specific $\mathrm{N}$ - and C-side distinct domain families adjacent to each domain. We found that most domains have one or two distinct adjacent domain families. The features of the obtained domain versatility are consistent with earlier reports that the number of different partner domains for a single domain or for a domain combination follows a power law distribution: many domains or domain combinations have only very few different $\mathrm{N}$-terminal or C-terminal partner domains. The most versatile domain in the three kingdoms is Ank (PF00023), having 220 distinct partner domain families in eukaryotes.

We calculated kingdom-specific IDF and IV scores for all domains using eq. 1 and 2, and obtained weight scores for each domain by the product of the IAF and IV scores. The scores were multiplied by 10 to facilitate computation. These domain's scores represent their importance in the protein universe and are used in the comparison of domain architectures. The analysis of the weight scores indicates that they are distributed 0.2 to 138.00 (Table 1), where most scores are over 100 and a small number of domains have scores below 20. Top ten domains with lower scores in the three Kingdoms are given in Table 2 and the weight scores distribution over all Pfam domains is given at the website.

We examined the weight scores of previously known promiscuous domains to identify relationship between weight scores and domain promiscuity. To do this, 215 eukaryotic promiscuous domains published by Basu et al. [14] were used. These promiscuous domains consist of 76 Pfam domains and 139 Smart domains, and are involved in protein-protein interaction and have crucial roles interaction networks. To facilitate comparison between these known promiscuous domains and the weight scores, we converted the 139 Smart domains into the corresponding Pfam domains, where 108 Smart domains could be converted. We found that all of the

Table I: The distribution of weight scores across the three kingdoms of life

\begin{tabular}{lllll}
\hline Weight scores & Eukaryote & Bacteria & Archaea & Total \\
\hline $120-140$ & 3,767 & 4,430 & - & 8,197 \\
$100-120$ & 1,172 & 1,111 & 6,728 & 9,011 \\
$80-100$ & 1,229 & 971 & 825 & 3,025 \\
$60-80$ & 66 & 259 & 666 & 991 \\
$40-60$ & 820 & 441 & 104 & 1,365 \\
$20-40$ & 741 & 628 & 282 & 1,651 \\
$0-20$ & 980 & 935 & 170 & 2,085 \\
\hline
\end{tabular}

Table 2: Top-ten promiscuous domains in the three Kingdoms Numbers in parenthesis is the weight scores of Pfam domains

\begin{tabular}{llll}
\hline Order & Eukaryote & Bacteria & Archaea \\
\hline I & Ank (0.19) & TPR_2 (0.4I) & Fer4 (0.86) \\
2 & WD40 (0.24) & Response_reg (0.45) & PKD (I.7I) \\
3 & zf-C2H2 (0.3) & ABC_tran (0.47) & CBS (I.82) \\
4 & zf-C3HC4 (0.3) & AcetyltransfII (0.50) & Radical_SAM (2.I5) \\
5 & RRM_I (0.4I) & Fer4 (0.62) & AAA (2.50) \\
6 & 7tm_I (0.44) & TPR_I (0.63) & Response_reg (2.79) \\
7 & PH (0.46) & HATPase_c (0.64) & HATPase_c (2.8I) \\
8 & efhand (0.46) & fn3 (0.73) & HTH_5 (2.84) \\
9 & EGF (0.48) & HTH_3 (0.74) & PAS (3.08) \\
10 & MFS_I (0.53) & HisKA (0.75) & TPR_2 (3.I5) \\
\hline
\end{tabular}

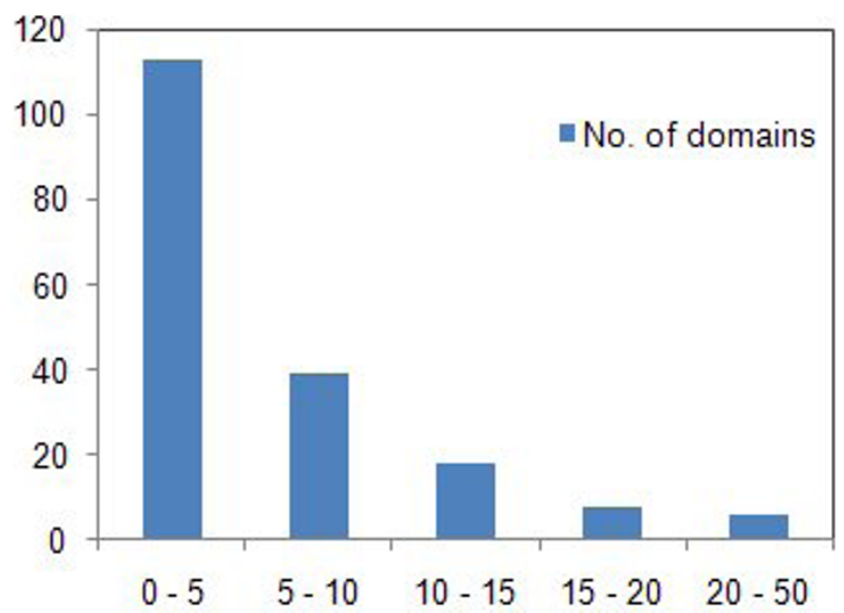

\section{Figure 4}

The distribution of weight scores of 215 eukaryotic promiscuous domains. All of the known promiscuous domains have very low weight scores, I 52 (83\%) mostly below 10. It means that the calculated weight scores represent domain promiscuity and importance of protein domains.

known promiscuous domains have very low weight scores, 152 (83\%) mostly below 10 (Figure 4). It means that the calculated weight scores represent domain promiscuity and importance of protein domains.

\section{Performance evaluation}

To assess the effect of domain weight scores on domain architecture comparison, the WDAC (weighted method) was compared to the general unweighted domain architecture comparison (DAC) method using all complete Homo sapiens (human) and Caenorhabditis elegans (nematode) protein sequences. In the DAC method, domain weight scores are not considered. To implement the DAC method, we used Jaccard similarity [26], which is commonly used in information retrieval, instead of the measure of cosine similarity used in the WDAC 
method. The Jaccard similarity can be calculated by the following equation:

$$
J(X, Y)=\frac{f_{11}}{f_{01}+f_{10}+f_{11}}
$$

where $f_{11}$ is the number of domains common to both sequences $\mathrm{X}$ and $\mathrm{Y}, f_{10}$ is the number of domains in $\mathrm{X}$, and $f_{01}$ is the number of domains in $\mathrm{Y}$.

We extracted all complete human and nematode protein sequences from RefSeq proteins, yielding 32,999 human and 23,220 nematode protein sequences. Among these proteins, 23,295 human and 14,522 nematode proteins have detectable Pfam domain information. Among the human proteins, we selected 9,764 proteins that contain more than 2 Pfam domains and performed domain architecture comparisons between the selected human proteins ( $\geq$ two domains) and those from the nematode proteome ( $\geq$ one domain) using the WDAC and DAC algorithms.

To validate homologous pairs of human and nematode proteins, we used the HomoloGene database [27], a NCBI dataset that curates sets of orthologs from the annotated genes of several completely sequenced eukaryotic genomes. Among the 44,481 groups in HomoloGene release 61 , we selected 2,559 groups that have both the selected human proteins and nematode proteins. From the comparison results, we extracted the WDAC and DAC results that have the same HomoloGene
ID in the query (human) and the best matched protein (nematode). The results show that the number of true positive values in the WDAC and DAC results are 2,328 (91\%) and 2,175 (85\%) respectively, which means that considering weight scores in domain architecture comparison can improve homology identification.

In addition, we found that the WDAC results have more specific homologs than the DAC results. Figure 5 shows the query results of a human protein NP_006695 (suppressor of the G2 allele of SKP1 isoform b). The best matched protein from the WDAC results is NP_080750 (SGT1, suppressor of the G2 allele of SKP1), while DAC results have two proteins, NP_080750 and NP_033916, as the best matched protein. The reason that DAC cannot discriminate between the two proteins is that DAC treats two domains, TPR_1 and Siah, equally.

\section{Construction of web server}

The query interface accepts protein sequences in FASTA format, and the maximum number of input protein sequences for a single submission is 100 and the length of each sequence is limited to 5000 residues. The output of the server is an HTML-formatted file, which consists of three parts: query domain architecture with Pfam domains, matched domain architecture, and domain information (Figure 6). For more than two sequences, users must input an email address to receive the WDAC results.

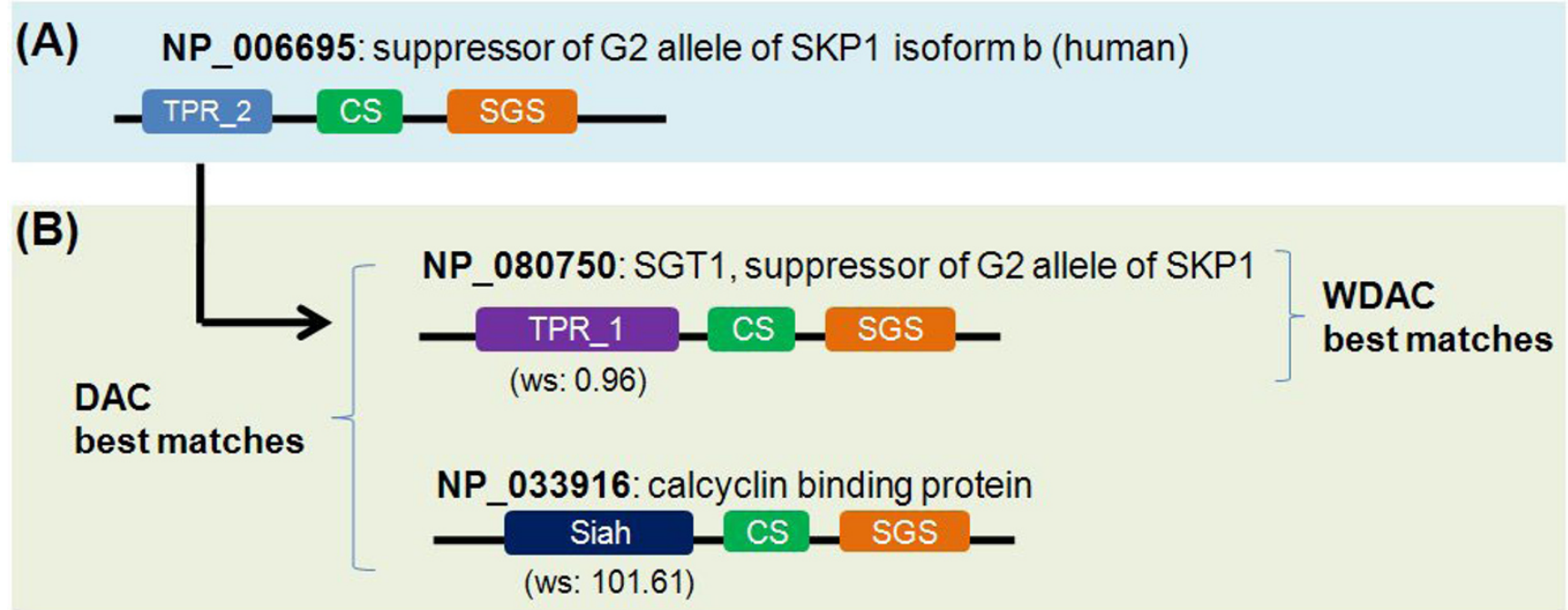

Figure 5

The best matches of the WDAC and DAC results for a human protein, NP_006695. (A) Query protein (human). (B) The best-matched proteins in the WDAC and DAC results. DAC cannot distinguish between two proteins (NP_080750 and NP_033916), while WDAC can identify more homologous proteins by using weight scores. 


\section{(A) 1) Domain architecture of a query protein}

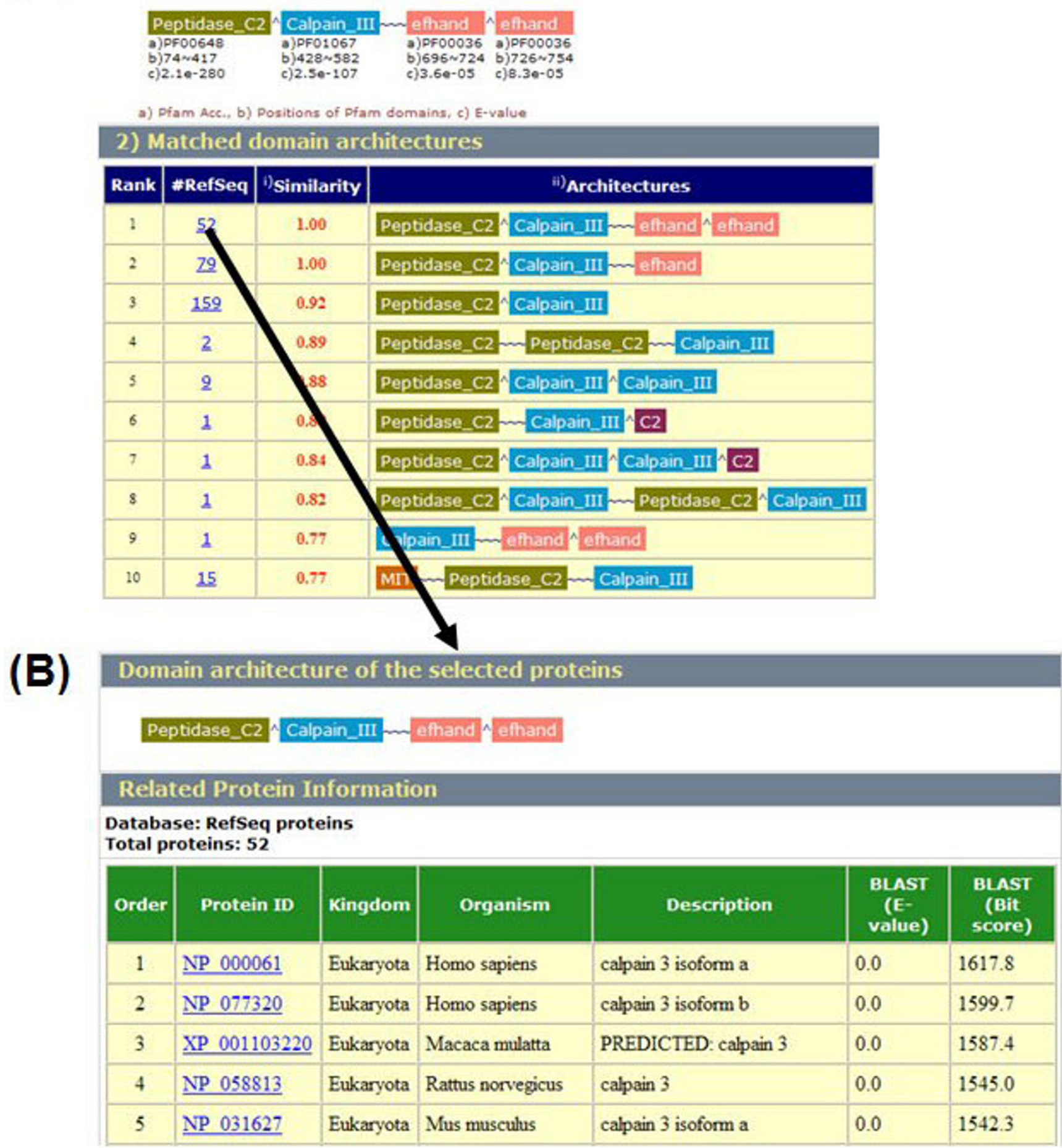

Figure 6

Screenshot of the results of the WDAC server for a query protein. (A) Matched domain architecture. (B) List of protein belonging to the selected domain architecture. 


\section{Conclusion}

There are several current homology methods which compare domain architectures. However, these methods are challenged by large families defined by promiscuous domains. To cope with the promiscuous domain problem, we present a method for measuring the similarity among protein domain architectures based on their Pfam-A domain annotations. The Pfam database may contain a small number of false positives and false negatives. Nevertheless, it is currently one of the most useful and practical domain annotation databases for protein sequences. In this study, we consider domain weight scores, obtained based on the abundance and versatility of domains. Our analysis indicates that considering domain weight scores in domain architecture comparison improves the performance of protein homology identification. The WDAC algorithm is also effective in resolving some issues that have baffled traditional sequence-based comparison methods, such as the comparison of proteins with promiscuous domain (s). The WDAC algorithm and its web server could be used to explore the underlying evolutionary relationships among proteins at the level of their whole domain architectures, rather than at the single-domain or protein sequence level.

\section{Competing interests}

The authors declare that they have no competing interests.

\section{Authors' contributions}

$\mathrm{BL}$ designed the algorithm, carried out the programming, analyzed the results and wrote the manuscript. DL directed the entire study. All authors read and approved the final manuscript.

\section{Note}

Other papers from the meeting have been published as part of BMC Genomics Volume 10 Supplement 3, 2009: Eighth International Conference on Bioinformatics (InCoB2009): Computational Biology, available online at http://www.biomedcentral.com/1471-2164/10? issue $=\mathrm{S} 3$.

\section{Acknowledgements}

BL was supported by the KRIBB Research Initiative Program and by the Korea Science and Engineering Foundation (KOSEF) grant funded by the Korea government (MEST) (No. MI0869030002-08N6903-00210). DL was supported by the Ministry of Knowledge Economy, Korea, under the ITRC support program supervised by the IITA (IITA-2008-CI090-080I000I) and Korea Institute of Science and Technology Information Supercomputing Center.

This article has been published as part of BMC Bioinformatics Volume 10 Supplement 15, 2009: Eighth International Conference on Bioinformatics (InCoB2009): Bioinformatics. The full contents of the supplement are available online at http://www.biomedcentral.com/|47/-2/05//0? issue $=S I 5$.

\section{References}

I. Song N, Sedgewick RD and Durand D: Domain architecture comparison for multidomain homology identification. J Comput Biol 2007, I4(4):496-5I6.

2. Punta $M$ and $O$ fran $Y$ : The rough guide to in silico function prediction, or how to use sequence and structure information to predict protein function. PLoS Comput Biol 2008, 4(10): el 000160.

3. Ponting CP and Russell RR: The natural history of protein domains. Annu Rev Biophys Biomol Struct 2002, 31:45-7I.

4. Lee B, Hong T, Byun SJ, Woo T and Choi YJ: ESTpass: a webbased server for processing and annotating expressed sequence tag (EST) sequences. Nucleic acids research 2007, 35 Web Server: W159-162.

5. Lee B and Shin G: CleanEST: a database of cleansed EST libraries. Nucleic acids research 2009, 37 Database: D686-689.

6. Song N, Joseph JM, Davis GB and Durand D: Sequence similarity network reveals common ancestry of multidomain proteins. PLoS Comput Biol 2008, 4(4): 1000063.

7. Hollich V and Sonnhammer EL: PfamAlyzer: domain-centric homology search. Bioinformatics (Oxford, England) 2007, 23 (24):3382-3383.

8. Chothia C, Gough J, Vogel C and Teichmann SA: Evolution of the protein repertoire. Science 2003, 300(5626): $1701-1703$.

9. Lin $K$, Zhu $L$ and Zhang DY: An initial strategy for comparing proteins at the domain architecture level. Bioinformatics (Oxford, England) 2006, 22(I7):208I-2086.

10. Tordai H, Nagy A, Farkas K, Banyai L and Patthy L: Modules, multidomain proteins and organismic complexity. The FEBS journal 2005, 272(19):5064-5078.

II. Fong JH, Geer LY, Panchenko AR and Bryant SH: Modeling the evolution of protein domain architectures using maximum parsimony. Journal of molecular biology 2007, 366(I):307-3I5.

12. Geer LY, Domrachev M, Lipman DJ and Bryant SH: CDART: protein homology by domain architecture. Genome research 2002, I 2(10):1619-1623.

13. Bjorklund AK, Ekman D, Light S, Frey-Skott $J$ and Elofsson A: Domain rearrangements in protein evolution. Journal of molecular biology 2005, 353(4):911-923.

14. Basu MK, Carmel L, Rogozin IB and Koonin EV: Evolution of protein domain promiscuity in eukaryotes. Genome research 2008, I 8(3):449-46I.

15. Lee $B$ and Lee D: DAhunter: a web-based server that identifies homologous proteins by comparing domain architecture. Nucleic Acids Res 2008, 36 Web Server: W60-64.

16. Basu MK, Poliakov E and Rogozin IB: Domain mobility in proteins: functional and evolutionary implications. Brief Bioinform 2009, I0(3):205-216.

17. Pruitt KD, Tatusova $T$ and Maglott DR: NCBI reference sequences (RefSeq): a curated non-redundant sequence database of genomes, transcripts and proteins. Nucleic acids research 2007, 35 Database: D6I-65.

18. Finn RD, Tate J, Mistry J, Coggill PC, Sammut SJ, Hotz HR, Ceric G, Forslund K, Eddy SR and Sonnhammer EL, et al: The Pfam protein families database. Nucleic acids research 2008, 36 Database: D28I-288.

19. Greene LH, Lewis TE, Addou S, Cuff A, Dallman T, Dibley M, Redfern O, Pearl F, Nambudiry R and Reid A, et al: The CATH domain structure database: new protocols and classification levels give a more comprehensive resource for exploring evolution. Nucleic acids research 2007, 35 Database: D29I-297.

20. Andreeva A, Howorth D, Chandonia JM, Brenner SE, Hubbard TJ, Chothia $C$ and Murzin AG: Data growth and its impact on the SCOP database: new developments. Nucleic acids research 2008 , 36 Database: D419-425.

21. Vogel C, Teichmann SA and Pereira-Leal J: The relationship between domain duplication and recombination. Journal of molecular biology 2005, 346(I):355-365.

22. Yu S, Van Vooren S, Tranchevent LC, De Moor B and Moreau Y: Comparison of vocabularies, representations and ranking algorithms for gene prioritization by text mining. Bioinformatics (Oxford, England) 2008, 24(16): : I I 9-125.

23. Glenisson P, Coessens B, Van Vooren S, Mathys J, Moreau $Y$ and De Moor B: TXTGate: profiling gene groups with text-based information. Genome Biol 2004, 5(6):R43. 
24. Altschul SF, Madden TL, Schaffer AA, Zhang J, Zhang Z, Miller W and Lipman DJ: Gapped BLAST and PSI-BLAST: a new generation of protein database search programs. Nucleic Acids Res 1997, 25(I 7):3389-3402.

25. Rattei T, Tischler P, Arnold R, Hamberger F, Krebs J, Krumsiek J, Wachinger B, Stumpflen $V$ and Mewes W: SIMAP-structuring the network of protein similarities. Nucleic Acids Res 2008, 36 Database: D289-292.

26. Balestre M, Von Pinho RG, Souza JC and Lima JL: Comparison of maize similarity and dissimilarity genetic coefficients based on microsatellite markers. Genet Mol Res 2008, 7(3):695-705.

27. Sayers EW, Barrett T, Benson DA, Bryant SH, Canese $K$, Chetvernin V, Church DM, DiCuccio M, Edgar R and Federhen S, et al: Database resources of the National Center for Biotechnology Information. Nucleic Acids Res 2009, 37 Database: D5-15.

Publish with BioMed Central and every scientist can read your work free of charge

"BioMed Central will be the most significant development for disseminating the results of biomedical research in our lifetime. "

Sir Paul Nurse, Cancer Research UK

Your research papers will be:

- available free of charge to the entire biomedical community

- peer reviewed and published immediately upon acceptance

- cited in PubMed and archived on PubMed Central

- yours - you keep the copyright 